\title{
Congenital infections as contributors to the onset of diabetes in children: A longitudinal study in the United States, 2001-2017
}

\author{
Mary A. M. Rogers ${ }^{1,2}$ ( ) | Catherine Kim ${ }^{1,2}$
}

${ }^{1}$ Department of Internal Medicine, University of Michigan, Ann Arbor, Michigan

${ }^{2}$ Department of Obstetrics \& Gynecology, University of Michigan, Ann Arbor, Michigan

\section{Correspondence}

Research Associate Professor Mary A. M. Rogers, Department of Internal Medicine, University of Michigan, Building 16, Room 422 W, North Campus Research Complex, 2800 Plymouth Road, Ann Arbor, MI 48109-2800.

Email: maryroge@umich.edu

\section{Peer Review}

The peer review history for this article is available at https://publons.com/publon/10. 1111/pedi.12957.

\begin{abstract}
Background: Maternal infections during pregnancy, particularly with rubella virus, were reported to increase the risk of diabetes in children. Widespread vaccination has decreased the number of infants with congenital rubella syndrome in the United States, although it remains a problem in developing countries. Because vaccine hesitancy has recently increased, we investigated the association between congenital infections with subsequent diabetes risk in children in the United States.

Methods: Using data from a nationwide private health insurer for years 2001-2017, 1475587 infants were followed for an average of 3.9 years (maximum 16.5 years). Information was obtained regarding congenital infections (rubella, cytomegalovirus, other congenital infections) and perinatal infections, as well as for the development of diabetes mellitus and diabetic ketoacidosis.

Results: There were 781 infants with congenital infections and 73974 with perinatal infections. Diabetes developed in 3334 children. The odds of developing diabetes for infants with congenital rubella infection were 12 -fold greater $(P=.013)$ and, for infants with congenital cytomegalovirus infection, were 4-fold greater $(P=.011)$ than infants without congenital or perinatal infection. Infants with other congenital infections had 3-fold greater odds of developing diabetes $(P=.044)$. Results were similar for diabetes ketoacidosis. Infants with other perinatal infections had $49 \%$ greater odds of developing diabetes during the follow-up period $(P<.001)$.

Conclusion: Congenital and other perinatal infections are associated with elevated risks of developing diabetes mellitus during childhood. Vaccination for rubella remains an important preventive action to reduce the incidence of diabetes in children.

KEYWORDS

congenital infection, cytomegalovirus, diabetes, rubella
\end{abstract}

\section{1 | INTRODUCTION}

Prior to widespread vaccination, maternal infections during pregnancy, particularly with rubella virus, were reported to increase the risk of diabetes in children. ${ }^{1}$ Long-term observation of individuals with congenital rubella syndrome demonstrated that approximately $22 \%$ developed diabetes by 60 years of age. ${ }^{2}$ In a cohort study of children from New York, $12.4 \%$ of the 242 children with congenital rubella 
syndrome developed diabetes by 17 years of age. ${ }^{3}$ Given a background rate of approximately 22 incident cases of diabetes per 100000 children per year in the United States, ${ }^{4}$ one child would have been expected to develop type 1 diabetes by age 17 in the New York cohort, instead of the 30 cases that actually occurred. ${ }^{3}$ The elevated rate of diabetes in children born with congenital rubella syndrome was often noted by physicians over the past half century. ${ }^{2}$

Congenital rubella infection remains an important public health issue in many countries and vaccination efforts to prevent rubella are ongoing. ${ }^{5}$ Not all congenital infections, however, can be prevented through vaccination and, with increased world travel, exposures during pregnancy remain a concern. ${ }^{6}$ Congenital cytomegalovirus infection, for example, occurs in both developed and developing countries and there is currently no vaccine, although vaccine development efforts are underway. ${ }^{7}$ Because vaccine hesitancy has increased over the past 20 years in the United States-particularly for the measles, mumps, rubella (MMR) vaccine, ${ }^{8}$ we investigated associations between infection, both congenital and perinatal, with subsequent diabetes risk in a longitudinal study in the United States between 2001 and 2017.

\section{2 | METHODS}

Data were from a nationwide private health insurer in the United States (Clinformatics DataMart Database, years 2001-2017). All infants with a minimum of 1 year of continuous insurance coverage were included, with the sole exclusion of infants with neonatal diabetes. Information was extracted regarding congenital rubella (ICD9771.0 or ICD-10 P35.0), congenital cytomegalovirus (ICD-9771.1 or ICD-10 P35.1), other congenital infections (ICD-9771.2 or ICD-10 P35.2, P37.0, P37.1, P37.3, P37.4, P37.8; listed as congenital herpes simplex, listeriosis, malaria, toxoplasmosis, or tuberculosis), and other perinatal infections (ICD-9771.3 to ICD-9771.8 or ICD-10 P35.2 to ICD-10 P35.9). Other perinatal infections were defined as acquired either in utero, during birth via the umbilicus, or during the first 28 days after birth. For purposes of this study and given the constraints of the ICD coding, we will refer to "other perinatal infections" as those infections acquired either in utero, during birth via the umbilicus, or during the first 28 days after birth-but excluding the congenital infections as categorized above (rubella, cytomegalovirus, herpes simplex, listeriosis, malaria, toxoplasmosis, tuberculosis). Because congenital rubella infection has been associated with both type 1 and type 2 diabetes, ${ }^{2,9}$ diabetes was determined using ICD-9 codes 249.x or 250.x, or ICD-10 codes E08.x or E10.x or E11.x. Diabetic ketoacidosis was identified by ICD-9 codes 250.1x, 250.3x and ICD10 codes E10.1x, E11.1x, and E13.1x. In sensitivity analyses, the sample was restricted to type 1 diabetes, using only ICD-9250.x1 or 250 . $\mathrm{x} 3$, or ICD-10 E10.x. Logistic regression was performed to assess the association between congenital infections and diabetes, with Stata/ MP 14.2 (StataCorp LLC, College Station, Texas). Two-sided P-values less than .05 were considered significant. This study was approved by the University of Michigan Institutional Review Board. Publicly available de-identified data were used and informed consent was not required.

\section{3 | RESULTS}

There were 1475587 infants who had insurance coverage for a minimum of 365 days (maximum 16.5 years). Infants were observed for a mean of 3.9 years $(S D=2.9)$. Of the infants, $48.6 \%$ were girls and $51.4 \%$ were boys.

There were 781 infants $(0.06 \%)$ who had congenital infections and 73974 (5.01\%) who had other perinatal infections. There were 38 infants with congenital rubella infection, 316 with congenital cytomegalovirus infection, and 427 infants with other congenital infections. During follow-up, diabetes occurred in 3334 children, $242(0.33 \%)$ who had a perinatal infection and seven $(0.90 \%)$ with congenital infections (one with rubella, three with cytomegalovirus, and three with other congenital infections), compared to 3085 $(0.22 \%)$ without congenital/perinatal infection $(P<.001)$.

In a logit model, the odds of developing diabetes for infants with congenital rubella infection were 12 -fold greater than those without (Table 1). Infants with congenital cytomegalovirus infection also had elevated odds of developing diabetes (odds ratio [OR] $=4.34$ ). Other congenital infections and other perinatal infections were also associated with diabetes mellitus (OR $=3.21$ and 1.50 , respectively). The risk difference (attributable risk) for congenital rubella syndrome was 24 per 1000 births in this study. The median time from birth to diabetes diagnosis for infants with perinatal infections (congenital and other perinatal infections combined) was 1.6 years and for infants without was 3 years.

There was also an association between diabetic ketoacidosis in children and a prior history of congenital rubella infection $(O R=12.41)$, congenital cytomegalovirus infection $(O R=4.40)$, other congenital infections ( $O R=3.25)$, as well as other perinatal infections $(\mathrm{OR}=1.50)$

Because rotavirus vaccination during infancy has been shown to lower rates of diabetes in childhood, ${ }^{10,11}$ the results were adjusted for completion of the rotavirus vaccine series (Table 1). After adjustment, congenital infections and other perinatal infections remained significantly associated with the incidence of diabetes. Of note, of the 38 infants with congenital rubella syndrome, six completed the rotavirus vaccine series and none developed diabetes. In the 32 infants with congenital rubella syndrome who did not complete the rotavirus vaccine series, one developed diabetes. Of the 316 infants with congenital cytomegalovirus infection, 92 completed the rotavirus vaccine series (none developed diabetes) and 224 did not complete the rotavirus vaccine series (three developed diabetes).

In sensitivity analysis, the OR for congenital rubella infection and type 1 diabetes was 23.53 (95\% Cl: 3.23, 171.62; $P=.002$ ). For congenital cytomegalovirus infection and type 1 diabetes, the OR was 8.35 (95\% Cl: 2.67, 26.04); $P<.001$ ). For other congenital infections, the OR was 2.04 (95\% Cl: $0.29,14.55 ; P=.475)$. For other perinatal 
TABLE 1 Congenital and perinatal infections as predictors of diabetes mellitus in children, United States, 2001-2017

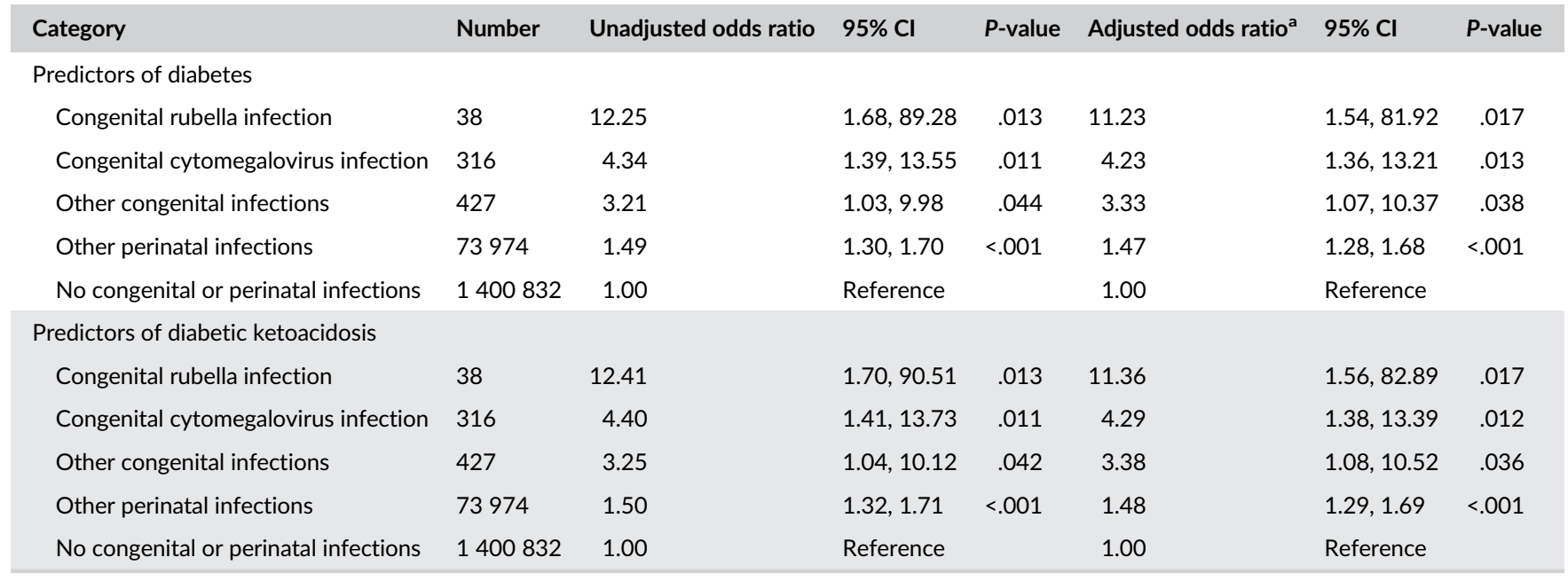

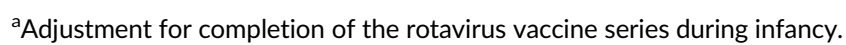

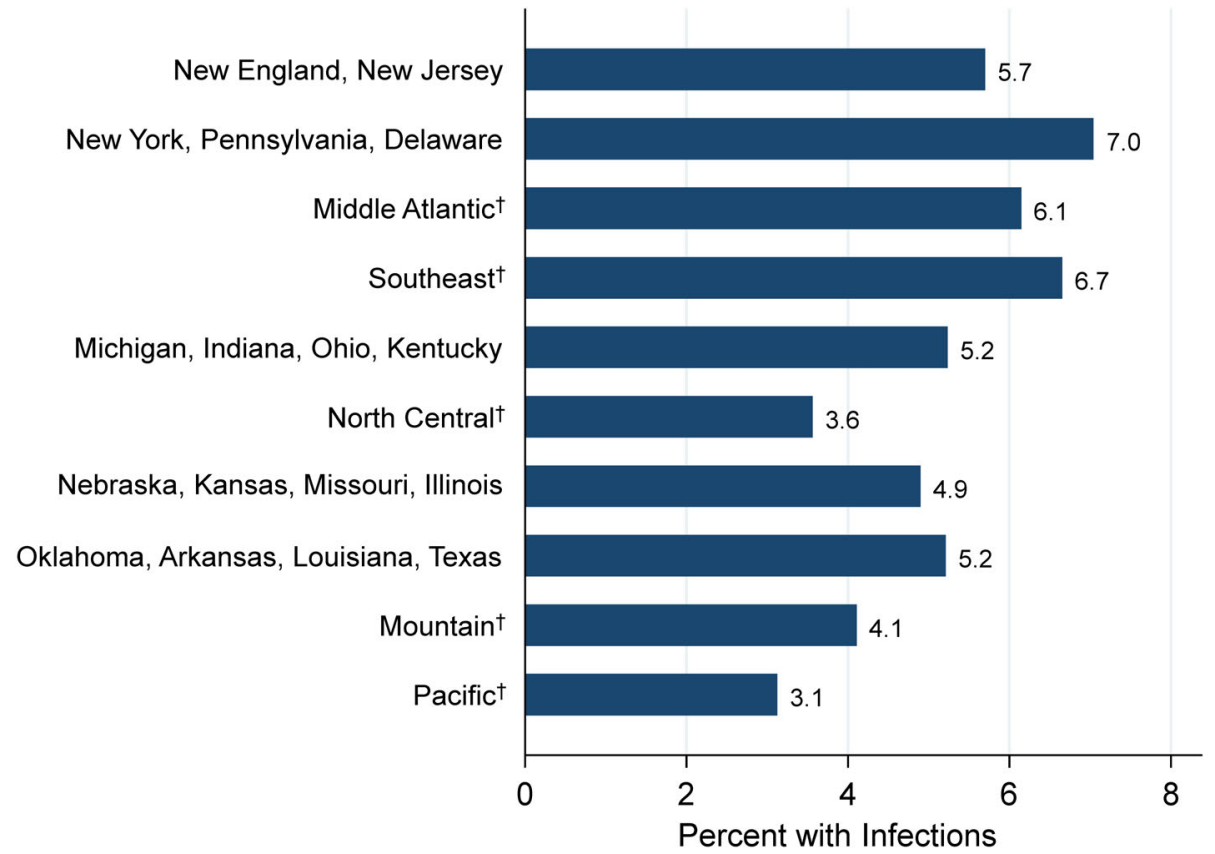

FIGURE 1 Percentage of infants with congenital and perinatal infections by location, United States, 2001-2017. †Middle Atlantic: Maryland, District of Columbia, Virginia, West Virginia, North Carolina, South Carolina. Southeast: Florida, Georgia, Alabama, Mississippi, Tennessee. North Central: Wisconsin, Minnesota, lowa, North Dakota, South Dakota, Montana. Mountain: Idaho, Wyoming, Colorado, Utah, Nevada, New Mexico, Arizona. Pacific: California, Oregon, Washington, Alaska, Hawaii infections and type 1 diabetes, the OR was 1.39 (95\% Cl: 1.15 , 1.68; $P=.001)$.

The frequency of congenital and perinatal infections (combined) varied significantly $(P<.001)$ across the country, from $7.0 \%$ in New York and Pennsylvania to $3.1 \%$ on the Pacific coast (Figure 1).

\section{DISCUSSION}

Congenital infections increase the risk of diabetes in the offspring. Congenital rubella infection was rare but associated with risk, as was cytomegalovirus infection. Perinatal infections, in general, were associated with diabetes and diabetic ketoacidosis in children in this study. Unfortunately, specific types of perinatal infections were only available for certain types of etiologic agents in this database. From previous research, various viruses have been implicated as triggers of islet autoimmunity and the onset of diabetes in children. ${ }^{12}$ In a systematic review of maternal viral infections in pregnancy, a metaanalysis of five case-control studies indicated a 2-fold increase in the risk of type 1 diabetes in the offspring when infected with enterovirus during pregnancy. ${ }^{13}$ To our knowledge, our study is the first cohort study to report the association between congenital viral infections and type 1 diabetes in children. Our findings affirm the previous research regarding the association between congenital rubella 
infection and type 1 diabetes, but also suggest that congenital cytomegalovirus infection and other perinatal infections could also influence risk.

Because live attenuated vaccines are contraindicated for pregnant women, our findings emphasize the importance of completing the rubella vaccine series at age-appropriate times to reduce the likelihood of transmission from the general public to pregnant women. Moreover, our results indicate that, among children with congenital infections, completion of the rotavirus vaccine series may be a pragmatic option to lower their risk of developing type 1 diabetes.

Screening for gestational diabetes and screening for type 2 diabetes in adults is currently recommended, but there is insufficient evidence for childhood diabetes screening in high risk groups. ${ }^{14,15}$ Our results suggest that screening in populations at high risk of diabetes, such as those with congenital rubella syndrome, should be evaluated. For children with a history of congenital infection, parental education regarding the symptoms of diabetic ketoacidosis is important. Diabetes ketoacidosis, common in children when first diagnosed, can be lethal and often results in hospitalization. ${ }^{16}$

In the United States, it is difficult to capture the total burden of congenital and perinatal infections because national surveillance is disease-specific, not all viral infections are under surveillance, and reporting criteria have changed over time. In our study, we found that perinatal infections (either in utero, during birth via the umbilicus, or during the first 28 days after birth) tended to occur more frequently in the eastern United States and ranged from 3.1\% to $7.0 \%$ of live births. As more large national databases become available, it is possible that additional information will be reported.

In conclusion, congenital and other perinatal infections are associated with the onset of diabetes mellitus in children. For some infections such as rubella, vaccination is available and, ${ }^{17}$ therefore, the benefits of vaccination should be emphasized in the population as a whole. Recognition of the early symptoms of type 1 diabetes is important for parents and guardians of children with a history of congenital infection.

\section{ORCID}

Mary A. M. Rogers (D) https://orcid.org/0000-0001-5519-3223

\section{REFERENCES}

1. Menser M, Forrest J, Bransby R. Rubella infection and diabetes mellitus. Lancet. 1978;311(8055):57-60.

2. Burgess MA, Forrest JM. Congenital rubella and diabetes mellitus. Diabetologia. 2009;52(2):369-370.
3. Ginsberg-Fellner F, Witt ME, Fedun B, et al. Diabetes mellitus and autoimmunity in patients with the congenital rubella syndrome. Clin Infect Dis. 1985;7(suppl 1):S170-S176.

4. Mayer-Davis EJ, Lawrence JM, Dabelea D, et al. Incidence trends of type 1 and type 2 diabetes among youths, 2002-2012. N Engl J Med. 2017;376(15):1419-1429.

5. Robertson SE, Featherstone DA, Gacic-Dobo M, Hersh BS. Rubella and congenital rubella syndrome: global update. Rev Panam Salud Publica. 2003;14:306-315.

6. McGovern LM, Boyce TG, Fischer PR. Congenital infections associated with international travel during pregnancy. J Travel Med. 2007; 14(2):117-128.

7. Rawlinson WD, Boppana SB, Fowler KB, et al. Congenital cytomegalovirus infection in pregnancy and the neonate: consensus recommendations for prevention, diagnosis, and therapy. Lancet Infect Dis. 2017;17(6):e177-e188.

8. Gostin LO, Ratzan SC, Bloom BR. Safe vaccinations for a healthy nation: increasing us vaccine coverage through law, science, and communication. JAMA. 2019;321(20):1969-1970.

9. Gale EA. Congenital rubella: citation virus or viral cause of type 1 diabetes? Diabetologia. 2008;51:1559-1566.

10. Perrett KP, Jachno K, Nolan TM, Harrison LC. Association of rotavirus vaccination with the incidence of type 1 diabetes in children. JAMA Pediatr. 2019;173(3):280-282.

11. Rogers MA, Basu T, Kim C. Lower incidence rate of type 1 diabetes after receipt of the rotavirus vaccine in the United States, 2001-2017. Sci Rep. 2019;9(1):7727.

12. Hyöty H. Viruses in type 1 diabetes. Pediatr Diabetes. 2016;17:56-64.

13. Allen DW, Kim KW, Rawlinson WD, Craig ME. Maternal virus infections in pregnancy and type 1 diabetes in their offspring: systematic review and meta-analysis of observational studies. Rev Med Virol. 2018;28(3):e1974.

14. Siu AL. Screening for abnormal blood glucose and type 2 diabetes mellitus: US preventive services task force recommendation statement. Ann Intern Med. 2015;163(11):861-868.

15. Moyer VA. Screening for gestational diabetes mellitus: US preventive services task force recommendation statement. Ann Intern Med. 2014;160(6):414-420.

16. Rewers A, Klingensmith G, Davis C, et al. Presence of diabetic ketoacidosis at diagnosis of diabetes mellitus in youth: the search for diabetes in youth study. Pediatrics. 2008;121(5):e1258-e1266.

17. Salmon DA, Dudley MZ, Glanz JM, Omer SB. Vaccine hesitancy: causes, consequences and a call to action. Vaccine. 2015;33:D66-D71.

How to cite this article: Rogers MAM, Kim C. Congenital infections as contributors to the onset of diabetes in children: A longitudinal study in the United States, 2001-2017. Pediatr Diabetes. 2020;21:456-459. https://doi.org/10.1111/pedi. 12957 